\title{
Construção de indicadores-padrão para cooperativas agropecuárias de Mato Grosso do Sul
}

\author{
Construction of standard indicators for agricultural Cooperatives of Mato Grosso do Sul
}

\begin{abstract}
Resumo
Este estudo teve como objetivo desenvolver, através de índices-padrão, um referencial de comparação para os índices econômico-financeiros das cooperativas agropecuárias do Estado de Mato Grosso do Sul (MS). Trata-se de uma pesquisa quantitativa, descritiva, bibliográfica e documental. Dessa forma, utilizou-se de balanços patrimoniais e demonstrações de sobras e perdas de dezoito cooperativas agropecuárias de Mato Grosso do Sul. A partir desses demonstrativos, foram calculados os indicadores econômico-financeiros dessas entidades, que receberam tratamento estatístico, com separação em quartis e definição da mediana, para se chegar aos índicespadrão. Com isso, foi possível classificar os índices econômico-financeiros das cooperativas, em excelentes, bons, regulares e insatisfatórios, se comparados com o padrão setorial. 0 trabalho se justifica pela relevância dos indicadores-padrão como base de avaliação do desempenho econômico-financeiro tanto pelas cooperativas como pelo Sistema OCB/MS.
\end{abstract}

Palavras-chave: Cooperativa. agronegócio. análise financeira. padrão setorial.

\begin{abstract}
This study aimed to develop, through standard indexes, a benchmark for the economic-financial indices of agricultural cooperatives in the State of Mato Grosso do Sul (MS). This is a quantitative, descriptive, bibliographical and documentary research. Accordingly, balance sheets and statements of leftovers and losses of eighteen agricultural cooperatives in Mato Grosso do Sul were used. From these statements, the economicfinancial indicators of these entities were calculated, which received statistical treatment, with quarantine and definition of the median, to arrive at the standard indexes. With this, it was possible to classify the economicfinancial indexes of the cooperatives, in excellent, good, regular and unsatisfactory, if compared with the sectorial standard. The work is justified by the relevance of the standard indicators as a basis for assessing economic and financial performance by both cooperatives and the OCB / MS System.
\end{abstract}

Keywords: Cooperative. agribusiness. financial analysis. sectorial standards.

Jose Aparecido Moura AranhaI', Laísa Aparecida Pereira de Souza ${ }^{I I}$, Agnes Akemi Yahiro ${ }^{I I I}$

IFundação Universidade Federal de Mato Grosso do Sul - UFMS. jaranha@terra.com.br

IIFundação Universidade Federal de Mato Grosso do Sul - UFMS. laisa_ln@hotmail.com

IIIFundação Universidade Federal de Mato Grosso do Sul - UFMS. agnesyahiro@gmail.com 


\section{Introdução}

O Produto Interno Bruto - PIB do Brasil apresentou crescimento de 1\% no primeiro trimestre de 2017, se comparado com o último trimestre de 2016, queda de 0,4\% se comparado com igual período de 2016, e decréscimo de 2,3\% em relação aos 4 últimos trimestres. Em 2018 o desempenho do PIB foi de crescimento de 1,1\% frete a 2017 (IBGE, 2019). Apesar de relativa estagnação do PIB, a agropecuária cresceu 15,2\% no primeiro trimestre de 2017, em comparação com igual período de 2016 (IBGE, 2017). No primeiro trimestre de 2018 a participação da agropecuária na formação do PIB pela ótica da produção foi de $6,1 \%$ e a representatividade do agronegócio nas exportações foi de 47,6\% em 2019 (MAPA, 2019).

Conforme os últimos dados publicados pela Secretaria de Estado de Meio Ambiente e Desenvolvimento Econômico - SEMADE (2016), a economia do MS cresceu 6\% em 2012, 6,6\% em 2013 e 2,6\% em 2014, com projeções de crescimento mínimo de $4 \%$ até 2021 , sendo válido destacar o notável desempenho do agronegócio sul-mato-grossense no cenário nacional e internacional, já que a agropecuária contribuiu expressivamente para o melhor desempenho do PIB do Mato Grosso do Sul, e representou em $2014,17,3 \%$ do mesmo.

Sabe-se que as cooperativas agropecuárias assumem um papel relevante no cenário supracitado, uma vez que contribuem para o desenvolvimento econômico e social, pois além da participação econômica, devido ao volume de produção local e de exportações, ainda propiciam a geração de milhares de empregos (LOPES; MENEZES, 2006).

Contudo, apesar das oportunidades que a globalização traz, como a participação da economia nacional no comércio internacional, conforme citado por Gimenes e Gimenes (2008), a competitividade e complexidade do mercado no qual as organizações estão inseridas, representam um grande desafio de vantagem competitiva para as empresas, inclusive para as cooperativas (MUNARETTO; CORRÊA, 2016).

0 mercado está mais exigente, os consumidores tornaram-se mais rigorosos, buscando sempre o melhor custo-benefício, a tecnologia está cada vez mais presente também no agronegócio e as exigências legais quanto à qualidade dos alimentos estão mais acentuadas. Como consequência, as cooperativas precisam adaptar-se a esse mercado, buscando estratégias para atrair o capital de seus membros, e para organizarem sua produção, sobrevivendo à competitividade do mercado e melhorando seu desempenho financeiro (COOK; CHADDAD, 2004 apud KALOGERAS et al., 2013).

Diante da inserção desse setor na economia, faz-se necessário desenvolver uma boa gestão econômico-financeira do agronegócio, pois segundo Corrêa, Kliemann Neto e Denicol (2016), o avanço do agronegócio se inter-relaciona com o uso eficiente dos recursos naturais, técnicos, humanos, e com enfoque especial neste trabalho - dos recursos financeiros.

Nesse contexto, destaca-se a contabilidade como importante ferramenta de gestão e fonte de fornecimento de informações para as entidades, informações essas que quando relevantes, auxiliam os tomadores de decisões internos e externos. Contudo, as empresas utilizam não só a própria contabilidade para tomar decisões, mas também têm utilizado instrumentos financeiros e econômicos como forma de comparação entre si, pois a comparabilidade das informações contribui para o processo de tomada de decisão (DUARTE; LAMOUNIER, 2007).

Além disso, como consequência da globalização, as entidades passaram a adotar os padrões internacionais de contabilidade, o que representa uma oportunidade para as entidades aperfeiçoarem a qualidade da informação contábil, além de torná-la mais comparável (RENDÓN-ÁLVAREZ; RODRÍGUEZ-BOLAÑOS; RIASCOS-GÓMEZ, 2013).

Devido à relevância do agronegócio para a economia do Brasil e à grande disponibilidade de recursos naturais no País, é necessário que haja estudos sobre esse setor, abrangendo principalmente o cooperativismo, a fim de que haja uma melhora crescente na gestão dos recursos, no aumento da produtividade e no desempenho social e financeiro (MOREIRA; BARREIROS; PROTIL, 2011).

No entanto, almejar bom desempenho financeiro, observando apenas os resultados obtidos pela própria cooperativa é limitador, sobretudo quando o mercado se mostra cada vez mais 
competitivo. Desta forma, a questão de pesquisa do presente estudo é: Como desenvolver, a partir de indicadores econômico-financeiros, índices-padrão para cooperativas agropecuárias de Mato Grosso do Sul?

Nessa perspectiva, o objetivo geral da presente pesquisa é desenvolver, a partir de indicadores econômico-financeiros, índices-padrão como referencial de comparação para as cooperativas agropecuárias do Estado de Mato Grosso do Sul. Já os objetivos específicos são: a) Desenvolver um padrão de comparação para cooperativas agropecuárias, utilizando os indicadores econômicofinanceiros, capaz de proporcionar uniformidade aos dados; b) avaliar o desempenho das cooperativas agropecuárias do Estado de Mato Grosso do Sul, de acordo com os índices-padrão do segmento.

Cabe ressaltar que os indicadores econômico-financeiros utilizados foram os tradicionais de que tratam a vasta literatura, entretanto estes podem ser facilmente aplicados em organizações cooperativas.

Segundo dados do relatório de 2016 da Organização das Cooperativas do Brasil (OCB, 2016), $48 \%$ de toda produção agrícola brasileira passa, de alguma maneira, por uma cooperativa agropecuária, o que evidencia que as cooperativas possuem peso considerável no agronegócio. A relevância e contribuição do presente estudo está na possibilidade de comparação entre cooperativas do ramo agropecuário, pois através da avaliação de desempenho por meio de um referencial padrão, as cooperativas terão parâmetros quantitativos para aprimorarem seu desenvolvimento e contribuir de forma ainda mais representativa para a economia do estado de Mato Grosso do Sul e consequentemente do País. Dessa forma os indicadores-padrão construídos podem ser utilizados para comparação da situação econômico-financeira pelas cooperativas agropecuárias do Estado bem como pelo Sistema OCB/MS.

0 trabalho estrutura-se em cinco seções. Na primeira, apresenta-se a contextualização do agronegócio no estado do Mato Grosso do Sul, além da exposição da justificativa da pesquisa e dos objetivos gerais e específicos. Posteriormente, é destacado o embasamento teórico da pesquisa. Na terceira seção, ocorre a caracterização da metodologia adotados. Já na quarta parte, os resultados são analisados e discutidos, através de gráficos e tabelas. Para finalizar, apresentam-se as conclusões e as referências bibliográficas da pesquisa.

O presente estudo é resultado do Projeto de Pesquisa "Construção de Indicadores Setoriais para Cooperativas Agropecuárias e de Crédito no Estado de Mato Grosso do Sul”, com fomento pela Fundação de Apoio ao Desenvolvimento do Ensino, Ciência e Tecnologia do Estado de Mato Grosso do Sul - FUNDECT, chamada FUNDECT № 10/2015 - UNIVERSAL - MS.

\section{Referencial Teórico}

\subsection{Caracterização do Sistema Cooperativo}

O cooperativismo é um modelo de negócios marcado pela gestão democrática, onde todos os seus membros participam ativamente das decisões, a partir da associação livre de pessoas que se unem de forma voluntária para atender suas necessidades comuns, sejam elas econômicas, sociais ou culturais, por meio de empreendimento de propriedade comum, objetivando promover o desenvolvimento individual e coletivo de maneira sustentável (ORGANIZAÇÃO INTERNACIONAL DO TRABALHO, 2002; OCB, 2017).

Desde a formação de uma cooperativa há a intenção de promover uma melhoria social, agregando valores que não se restringem à lucratividade, tais como a autoajuda, solidariedade, responsabilidade, democracia e igualdade. Para que as cooperativas coloquem esses valores em prática, existem alguns princípios que são os pilares do cooperativismo, dentre os quais estão a adesão livre e voluntária; gestão democrática; participação econômica dos membros; autonomia e independência; educação, formação e informação; intercooperação; e interesse pela comunidade (INTERNATIONAL CO-OPERATIVE ALLIANCE, 2017).

Para Bento, Oliveira e Madruga (2016), a cooperativa é uma organização criada para prestar auxílio social e econômico aos seus associados que se dispõem voluntariamente a participarem desta, 
de modo que formem uma organização democrática. Ademais, para Carpes, Schons e Oliveira (2012), a pluralidade de áreas abrangidas pelo cooperativismo edifica um sistema de relevância econômico social, pois a presença deste nos diversos setores da economia pode proporcionar uma melhor distribuição de renda a determinados grupos.

A cooperativa tem como algumas de suas responsabilidades criar e promover atividades geradoras de renda e emprego decente e sustentável, fortalecer sua competitividade e desenvolver seu potencial comercial, inclusive suas capacidades empresariais e gerenciais. Com recomendações formuladas pela Organização Internacional do Trabalho, pode-se perceber características muito parecidas com uma empresa. Porém, a cooperativa apresenta uma faceta que vai além dos padrões das empresas, que agrega o sentido do cooperativismo.

É de concordância que as cooperativas possuem papel relevante na sociedade, e Almeida et al. (2009) corroboram com este pensamento, afirmando que a própria sociedade reconhece as contribuições relevantes do cooperativismo não só nas localidades em que as cooperativas estão instaladas, mas também em todo o sistema produtivo mundial.

Outra característica importante das cooperativas é que as mesmas não possuem como objetivo principal o lucro, mas devido à competitividade do mercado no qual estão inseridas, é extremamente importante que as cooperativas apresentem boa eficiência financeira, visto que o desempenho superavitário - denominado sobra - é que lhes garantem a perenidade, pois a sobra é reinvestida nas companhias ou distribuída aos seus associados, de acordo com suas atividades, possibilitando que estas continuem cumprindo seu papel social (CARVALHO et al., 2015). Reforçando a necessidade de uma gestão qualificada, Almeida et al (2009) afirmam que, muitas vezes, as cooperativas assumem características de empresas com fins lucrativos, de forma que o resultado superavitário é imprescindível.

Os objetivos e metas são traçados numa cooperativa e aprovados democraticamente pelos associados que delas participam. Desta forma, é desenvolvido de maneira sustentável a economia da região que se insere. Para que os objetivos e retornos esperados sejam alcançados, é imprescindível que essas entidades desenvolvam uma boa governança, além de adotarem a contabilidade como instrumento de gestão e controle na administração do patrimônio das cooperativas (CARPES; ORO, 2010).

É evidente que o desafio de gerir uma cooperativa está além de obstáculos gerenciais e de controle dos que ocorrem nas empresas, mas também devido aos interesses dos associados e sua influência no poder de decisão. Segundo Lauermann (2016), isso ocorre porque o associado pode ser ao mesmo tempo cliente, fornecedor, usuário, proprietário e gestor da cooperativa e isso implica em obstáculos com relação ao planejamento e controle, pois os interesses dos participantes, apesar de comuns, podem também ser conflitantes.

As cooperativas possuem muitas limitações, pois além desses conflitos de interesse, na maioria das vezes não possuem recursos suficientes para melhorar sua estrutura produtiva, nem a qualidade dos seus produtos, e a qualificação de seus membros e gestores. Contudo, essas restrições, sejam elas humanas ou financeiras, são agravadas quando existe a má gestão dos recursos (ROYER; BIJMAN; ABEBE, 2017).

Com relação à gerência de recursos, Bento, Oliveira e Madruga (2016), expressam que as pessoas, sejam elas físicas ou jurídicas para realizar um bom planejamento, buscam descobrir e minimizar os custos e despesas, de produção ou financeiro, com a finalidade de conseguir uma melhora nas receitas. Numa cooperativa, apesar de não haver o foco no lucro, Carpes e Oro (2010), afirmam que se faz necessário entender que uma boa gestão dos recursos, irão sustentar a evolução do patrimônio e consequentemente poderão atender integralmente o objetivo de seus cooperados.

\subsubsection{Cooperativismo Brasileiro e Sul-Matogrossense e o Agronegócio}

O movimento cooperativista iniciou-se oficialmente no Brasil em 1889, devido à fundação da Cooperativa Econômica dos Funcionários Públicos de Ouro Preto. Em 1969, foi criada a OCB, órgão responsável pela representação do cooperativismo brasileiro e dois anos depois, foi promulgada a Lei 
no 5.764/71, cujo Art. 5o expressa que as cooperativas "poderão adotar por objeto qualquer gênero de serviço, operação ou atividade" (OCB, 2017)

Atualmente as cooperativas se encontram em 13 setores da economia: agropecuário, consumo, crédito, educacional, especial, habitacional, infraestrutura, mineral, produção, saúde, trabalho, transporte e turismo e lazer. Porém, é o setor agropecuário que se destaca, abrangendo aproximadamente 1500 cooperativas, ou seja, 23\% do total do país, além de gerar mais de 188.000 empregos formais. No Mato Grosso do Sul, a realidade não é diferente, visto que as cooperativas agropecuárias representam $50 \%$ do total existente no Estado, e as demais estão distribuídas nos outros 12 setores da economia (OCB/MS, 2016).

0 agronegócio é uma das atividades mais relevantes para a economia brasileira, e com grande potencial de expansão (JANK; NASSAR; TACHINARDI, 2005). Tais afirmações podem ser confirmadas analisando-se o saldo da balança comercial das cooperativas, que até setembro de 2017, apresentou uma variação positiva de 19,36\% nas exportações brasileiras, cabendo salientar que o Mato Grosso do Sul é o quinto Estado com maior participação nas exportações do Brasil (MINISTÉRIO DA INDÚSTRIA E DO COMÉRCIO, 2017).

Evidentemente, a gestão das cooperativas de cada ramo diferencia-se conforme a área de atuação, a estrutura administrativa e organizacional, o conhecimento, a experiência, habilidades e atitudes de seus administradores, devendo este estudo trazer contribuições acerca da análise financeira utilizando índices-padrão de cooperativas agropecuárias, a fim de que as entidades, ao conhecerem a realidade do ramo, possam desenvolver mecanismos eficientes de gestão, e consequentemente buscar o desempenho financeiro e social.

\subsection{Análise do Desempenho Financeiro de Cooperativas Agropecuárias Utilizando Índices-Padrão}

A análise das demonstrações financeiras, realizada através do cálculo e interpretação de indicadores econômico-financeiros, objetiva medir o desempenho das empresas. Por isso, essa análise deve ocorrer frequentemente, a fim de que seja possível conhecer a realidade da empresa estimar suas futuras perspectivas, auxiliando os gestores a tomarem decisões acertadas (DIEL et al., 2014).

Ao realizar a comparação dos índices de determinada empresa, bem como a mensuração do seu desempenho, é possível analisar o comportamento dos resultados em vários períodos, verificando assim a eficiência financeira da entidade. (PEREIRA et al., 2009). Mas, por se tratar de um ambiente competitivo, a entidade precisa avaliar sua situação perante o setor, e, por isso, Aranha (2015, p. 152) enfatiza que "apenas através de comparação é possível avaliar se uma entidade se apresenta em situação melhor ou pior que as outras".

Diante dessa demanda, destaca-se o índice-padrão, que para Silva $(2013$, p. 325$)$ pode ser definido como "um referencial de comparação", isto é, um referencial que possibilita a comparação de um determinado índice econômico-financeiro de uma empresa específica com o índice-padrão, representado pela mediana, ou seja, o número que ocupa a posição central de toda a amostra, dividindo os indicadores entre maiores e menores que a medida central.

Matarazzo (2010) elenca uma sequência de procedimentos para que se calculem os índicespadrão, sendo o primeiro passo definir o ramo de atividade das empresas, logo após calcular os índices econômico-financeiros, colocá-los em ordem crescente de grandeza, e então o padrão será dado pelos decis ou quartis, dependendo do tamanho da amostra.

Os decis e quartis são medidas estatísticas utilizadas com propósito de apurar o valor-padrão, dividindo o rol de indicadores econômico-financeiros em nove e três partes, respectivamente. Desta forma, o quinto decil e o segundo quartil representam a mediana da totalidade dos valores calculados (ASSAF NETO, 2015). Para este trabalho, dado o tamanho da amostra, decidiu-se dividi-la em quartis. Isto posto, após cálculos dos indicadores econômico-financeiros para o ano de 2013, é possível determinar a mediana de cada índice, que representará o padrão, coincidindo com o segundo quartil, conforme consta na Tabela 1. 
Tabela 1 - Análise comparativa para amostras divididas em quartis

\begin{tabular}{ccccc}
\hline Índice & $\mathbf{1 0}$ quartil & $\mathbf{2}^{\mathbf{0}}$ quartil & 3 quartil & 4 quartil \\
\hline $\begin{array}{c}\text { Quanto maior, } \\
\text { melhor }\end{array}$ & Insatisfatório & Regular & Bom & Excelente \\
$\begin{array}{c}\text { Quanto menor, } \\
\text { melhor }\end{array}$ & Excelente & Bom & Regular & $\begin{array}{c}\text { Insatisfatóri } \\
\text { o }\end{array}$ \\
\hline
\end{tabular}

Fonte: Assaf Neto (2015).

Para Assaf Neto (2015, p. 278) "os índices são classificados em duas categorias, sendo elas, quanto maior, melhor e quanto menor, melhor". Por isso, sugere uma análise comparativa, utilizando quartis, de modo o qualificar os valores ocupantes de cada parte que a amostra foi dividida, conforme apresentado na tabela 01 .

A utilização de índices-padrão permite a comparabilidade entre entidades do mesmo setor, propiciando verificar, no caso estudado, como está a situação financeira das cooperativas agropecuárias do Mato Grosso do Sul.

\subsection{Estudos Correlatos}

A seguir, no quadro 1, referenciam-se alguns estudos com objetivos e temáticas similares às estudadas neste trabalho.

Quadro 1 - Pesquisas similares à temática

\begin{tabular}{|c|c|c|}
\hline Referências & Objetivo & $\begin{array}{c}\text { Índices } \\
\text { Utilizados }\end{array}$ \\
\hline $\begin{array}{c}\text { Gozer, } \\
\text { Gimenes, } \\
\text { Menezes, } \\
\text { Gozer e } \\
\text { Gimenes } \\
(2007) .\end{array}$ & $\begin{array}{l}\text { Avaliar o desempenho econômico-financeiro de uma cooperativa } \\
\text { agropecuária através da utilização de índices-padrão. }\end{array}$ & $\begin{array}{l}\text { Liquidez; } \\
\text { Estrutura de } \\
\quad \text { Capital; } \\
\text { Rentabilidade; }\end{array}$ \\
\hline $\begin{array}{l}\text { Duarte e } \\
\text { Lamounier } \\
\text { (2007). }\end{array}$ & $\begin{array}{l}\text { Desenvolver um estudo que permita a avaliação da situação } \\
\text { econômico-financeira de empresas de capital aberto do setor da } \\
\text { Construção Civil, por meio da comparação com índices-padrão. }\end{array}$ & $\begin{array}{l}\text { Liquidez; } \\
\text { Rentabilidade; } \\
\text { Dinâmica do } \\
\text { Capital de Giro. }\end{array}$ \\
\hline $\begin{array}{l}\text { Pereira, } \\
\text { Carvalho e } \\
\text { Parente } \\
\text { (2011). }\end{array}$ & $\begin{array}{l}\text { Analisar o desempenho econômico e a evidenciação ambiental das } \\
\text { oito empresas que apresentaram os melhores relatórios de } \\
\text { sustentabilidade, segundo a pesquisa Rumo à Credibilidade } 2010 .\end{array}$ & Rentabilidade. \\
\hline $\begin{array}{l}\text { Aranha } \\
\text { (2015). }\end{array}$ & $\begin{array}{l}\text { Construir um modelo de indicadores para o setor elétrico } \\
\text { brasileiro, ou seja, um método de comparação com base em } \\
\text { medianas setoriais. }\end{array}$ & $\begin{array}{c}\text { Liquidez; } \\
\text { Estrutura de } \\
\text { Capital; } \\
\text { Rentabilidade; } \\
\text { Dinâmica do } \\
\text { Capital de Giro; } \\
\text { Geração de } \\
\text { Capital; Îndices de } \\
\text { Rotação. }\end{array}$ \\
\hline $\begin{array}{l}\text { Silva, Mello, } \\
\text { Gonze e Orrico } \\
\text { Filho (2015). }\end{array}$ & $\begin{array}{l}\text { Estabelecer comparação econômico-financeira entre as empresas } \\
\text { que atuam no setor de exploração de Rodovias, listadas na } \\
\text { BM\&FBovespa, construindo um índice-padrão que possa } \\
\text { estabelecer uma comparação de desempenho setorial. }\end{array}$ & $\begin{array}{c}\text { Liquidez; } \\
\text { Estrutura de } \\
\text { Capital; } \\
\text { Rentabilidade; } \\
\text { Modelo de Kanitz. }\end{array}$ \\
\hline $\begin{array}{l}\text { Jahara, Mello e } \\
\text { Afonso (2016). }\end{array}$ & $\begin{array}{c}\text { Realizar uma análise financeira dos clubes que disputaram a série A do } \\
\text { campeonato brasileiro no ano de 2014, com base no estabelecimento de índice } \\
\text { padrão. }\end{array}$ & $\begin{array}{l}\text { Liquidez; } \\
\text { Estrutura de Capital; } \\
\text { Rentabilidade; }\end{array}$ \\
\hline
\end{tabular}




\begin{tabular}{|c|c|c|}
\hline $\begin{array}{c}\text { Rossés, } \\
\text { Ferreira, } \\
\text { Stecca e Gelatti } \\
(2011)\end{array}$ & $\begin{array}{l}\text { Analisar o sistema de gestão da Cooperativa Agropecuária Júlio de } \\
\text { Castilhos (COTRIJUC) }\end{array}$ & $\begin{array}{c}\text { Avaliar os } \\
\text { componentes: } \\
\text { estruturais, } \\
\text { estratégicos, } \\
\text { diretivos, } \\
\text { comportamentais, } \\
\text { tecnológicos, de } \\
\text { mudança e de } \\
\text { avaliação }\end{array}$ \\
\hline $\begin{array}{l}\text { Bialoskorski } \\
\quad \text { Neto }\end{array}$ & $\begin{array}{c}\text { Discutir os fatores que influenciam a participação dos associados } \\
\text { nas cooperativas agropecuárias e, em especial, nas assembleias } \\
\text { gerais e comitês educativos. }\end{array}$ & $\begin{array}{c}\text { Índice financeiro } \\
\text { de Kanitz; número } \\
\text { de associados } \\
\text { ativos; assistência } \\
\text { técnica oferecida, } \\
\text { produção agrícola } \\
\text { entregue e } \\
\text { proporção de } \\
\text { associados no } \\
\text { número de } \\
\text { produtores rurais }\end{array}$ \\
\hline
\end{tabular}

Fonte: Os autores (2019).

Nota-se que a análise do desempenho de empresas, utilizando índices-padrão foi objeto de vários estudos, porém, com exceção de Gozer et al (2007), abrangem setores alheios à agropecuária, outros que tratam de gestão cooperativa não abordam a temática da pesquisa, portanto, há uma lacuna sobre o tema abordado neste trabalho.

0 trabalho de Gozer et al (2007), apesar de objetivar avaliar o desempenho de apenas uma cooperativa agropecuária, utilizou para tal feito, os índices-padrão calculados por Gimenes (2006) em seu pós-doutoramento na Universidade de São Paulo (USP), abrangendo uma amostra de cooperativas agropecuárias brasileiras, contudo, refere-se a períodos anteriores ao abordado neste estudo.

\section{Metodologia}

A pesquisa possui abordagem quantitativa, pois segundo Zanella (2009) utiliza-se de instrumentos estatísticos para medir e quantificar os resultados. Também é caracterizada como descritiva, e quanto aos procedimentos, classifica-se em bibliográfica e documental, visto que se fundamenta em fontes bibliográficas, e em documentos que foram padronizados, tais como os balanços patrimoniais e demonstrações de sobras e perdas das cooperativas. (GIL, 2008).

Para realização deste trabalho, utilizou-se uma amostra de dezoito cooperativas agropecuárias de uma população de 36 entidades segundo a OCB-MS (2019). A seleção da amostra foi por se tratar das que disponibilizaram seus demonstrativos contábeis, bem como por serem as mais representativas em termos de faturamento. Entende-se que a amostra, pelo porte, faturamento, número de associados e segmento em que atuam podem ser tomadas como referência de comparação de desempenho. Convém salientar que para o desenvolvimento deste trabalho, optou-se por manter a discrição quanto aos nomes de cada cooperativa, e por isso, como forma de diferenciação, as mesmas foram identificadas através de letras, que vão de A até R.

As demonstrações contábeis obtidas foram padronizadas, seguindo um modelo disponibilizado pelo Observatório de Cooperativismo da FEA-RP/USP. Os valores das demonstrações contábeis foram atualizadas monetariamente, pois segundo Takamatsu e Lamounier (2006), quando se analisa valores de vários exercícios, a existência da inflação, mesmo que moderada, faz com que os valores envolvidos nas análises caracterizem-se como grandezas não uniformes, comprometendo a qualidade das demonstrações contábeis e consequentemente a tomada de decisão.

Os índices econômico-financeiros utilizados para a construção do padrão foram os tradicionais empregados para análise das organizações empresariais. A sua utilização está pautada pela ausência 
de indicadores consistentes para mensurar as especificidades da gestão financeiras das organizações cooperativas, portanto, há uma lacuna na literatura, porém, esse fato não invalida o resultado desta pesquisa.

Com base nas demonstrações contábeis, foram calculados índices econômico-financeiros, conforme descrito no quadro 2 a seguir:

Quadro 2 - Índices utilizados para a análise de desempenho econômico-financeiro das cooperativas agropecuárias do Mato Grosso do Sul

\begin{tabular}{|c|c|c|c|}
\hline GRUPO & ÍNDICE & FÓRMULA & INTERPRETAÇÃO \\
\hline SOLVÊNCIA & $\begin{array}{c}\text { Liquidez Corrente } \\
\text { Liquidez Seca } \\
\text { Liquidez Geral }\end{array}$ & $\begin{array}{c}\mathrm{AC} / \mathrm{PC} \\
(\mathrm{AC}-\text { Estoques }) / \mathrm{PC} \\
\mathrm{AC}+\mathrm{RLP} / \mathrm{PC}+\mathrm{ELP}\end{array}$ & $\begin{array}{c}\text { Quanto maior, } \\
\text { melhor }\end{array}$ \\
\hline RENTABILIDADE & $\begin{array}{l}\text { Rentabilidade do } \\
\text { Ativo } \\
\text { Rentabilidade do } \\
\text { Patrimônio Líquido } \\
\text { Giro do Ativo } \\
\text { Margem Líquida }\end{array}$ & $\begin{array}{c}\text { (Sobra e Perda } \\
\text { Operacional/Ativo) x } 100 \\
\text { (Sobra e Perda } \\
\text { Operacional/PL) x } 100 \\
\text { Vendas Líquidas/AT } \\
\text { (Sobra e Perda } \\
\text { Operacional/Vendas } \\
\text { Líquidas) x } 100\end{array}$ & $\begin{array}{l}\text { Quanto maior, } \\
\text { melhor }\end{array}$ \\
\hline $\begin{array}{c}\text { ESTRUTURA DE } \\
\text { CAPITAL }\end{array}$ & $\begin{array}{c}\text { Participação de } \\
\text { Capital de Terceiros } \\
\text { Composição do } \\
\text { Endividamento } \\
\text { Imobilização do } \\
\text { Patrimônio Líquido }\end{array}$ & $\begin{array}{c}{[(\mathrm{PC}+\mathrm{ELP}) / \mathrm{PL}] \times 100} \\
{[\mathrm{PC} /(\mathrm{PC}+\mathrm{ELP})] \times 100} \\
\text { (Imobilizado + Investimento } \\
\text { + Intangível/PL) x } 100\end{array}$ & $\begin{array}{l}\text { Quanto menor, } \\
\text { melhor }\end{array}$ \\
\hline $\begin{array}{l}\text { ÍNDICES DE } \\
\text { ROTAÇÃOO }\end{array}$ & $\begin{array}{l}\text { Prazo Médio de } \\
\text { Estoque } \\
\text { Prazo Médio de } \\
\text { Recebimento } \\
\text { Prazo Médio de } \\
\text { Pagamento } \\
\text { Ciclo Operacional } \\
\text { Ciclo Financeiro }\end{array}$ & $\begin{array}{c}\text { (Estoque/CPV) x } 360 \\
{[(\text { Clientes }+} \\
\text { Cooperados }) / \text { Receita Bruta }] \\
\text { x } 360 \\
\text { (Fornecedor + Cooperados a } \\
\text { pagar/Compras a Prazo) x } \\
360 \\
\text { PME + PMR } \\
\text { CO - PMP }\end{array}$ & $\begin{array}{l}\text { Quanto menor, } \\
\text { melhor } \\
\text { Quanto menor, } \\
\text { melhor } \\
\text { Quanto maior, } \\
\text { melhor } \\
\text { Quanto menor, } \\
\text { melhor } \\
\begin{array}{c}\text { Quanto menor, } \\
\text { melhor }\end{array}\end{array}$ \\
\hline $\begin{array}{l}\text { DINÂMICA DO } \\
\text { CAPITAL DE } \\
\text { GIRO }\end{array}$ & $\begin{array}{c}\text { Capital Circulante } \\
\text { Líquido } \\
\text { Necessidade Capital } \\
\text { de Giro } \\
\text { Saldo em Tesouraria }\end{array}$ & $\begin{array}{c}(\mathrm{AC}-\mathrm{PC}) / \mathrm{Ativo}^{*} 100 \\
(\mathrm{ACO}-\mathrm{PCO}) / \text { Ativo*100 }^{*} 100 \\
(\mathrm{ACF}-\mathrm{PCF}) / \text { Ativo*100 }^{*}\end{array}$ & $\begin{array}{l}\text { Quanto maior, } \\
\text { melhor } \\
\text { Quanto menor, } \\
\text { melhor } \\
\text { Quanto maior, } \\
\text { melhor }\end{array}$ \\
\hline
\end{tabular}

Fonte: Adaptado de Matarazzo (2010).

Os índices calculados foram dispostos em ordem crescente de grandeza, para possibilitar calcular a mediana de cada um deles. Logo após, a amostra foi dividida em quartis, possibilitando uma análise comparativa dos índices econômico-financeiros das cooperativas agropecuárias. Os índices calculados, integrantes do primeiro quartil, cuja interpretação é quanto maior, melhor, foram qualificados como insatisfatórios, os do segundo quartil como regular, bom se no terceiro e excelente 
os que compuseram o quarto quartil. Já para os índices de interpretação quanto menor, melhor, a qualificação foi excelente, bom, regular e insatisfatório, para os índices que se enquadraram no primeiro quartil, segundo, terceiro e quarto, respectivamente.

\section{Análise e Discussões de Resultados}

\section{1 Índices-padrão}

0 acesso aos demonstrativos financeiros das dezoito cooperativas agropecuárias de Mato Grosso do Sul permitiu o cálculo dos indicadores econômico-financeiros, conforme apresentado no Apêndice A. Após a devida tabulação em ordem crescente de cada índice do tipo quanto maior, melhor, e decrescente para os do tipo quanto menor, melhor, definiram-se os quartis, e posteriormente os índices-padrão, conforme apresentado na Tabela 2.

Tabela 2 - Índice-Padrão e média de cada índice financeiro

\begin{tabular}{|c|c|c|c|c|}
\hline Índices & 1o quartil & $\begin{array}{l}2^{\circ} \text { quartil } \\
\text { (Mediana) }\end{array}$ & 3o quartil & Média \\
\hline \multicolumn{5}{|l|}{ Solvência } \\
\hline Liquidez Corrente - LC & 1,12 & 1,53 & 2,30 & 2,06 \\
\hline Liquidez Seca - LS & 0,81 & 1,15 & 1,63 & 1,60 \\
\hline Liquidez Geral - LG & 1,07 & 1,18 & 2,30 & 1,94 \\
\hline \multicolumn{5}{|l|}{ Rentabilidade } \\
\hline Rentabilidade do Ativo - ROA (\%) & $-0,91$ & 1,50 & 2,86 & 2,14 \\
\hline Rentabilidade do Patrimônio Líquido - ROE (\%) & $-3,02$ & 3,67 & 12,57 & 4,81 \\
\hline Giro do Ativo - GA (dias) & 0,95 & 1,46 & 3,57 & 2,75 \\
\hline Margem Líquida - ML (\%) & $-0,11$ & 1,26 & 4,43 & 1,65 \\
\hline \multicolumn{5}{|l|}{ Endividamento (\%) } \\
\hline Participação de Capital de Terceiros - PCT & 336,89 & 98,00 & 23,02 & 264,01 \\
\hline Composição do Endividamento - CE & 99,88 & 82,25 & 67,79 & 81,73 \\
\hline Imobilização do Patrimônio Líquido - IPL & 86,92 & 57,01 & 9,09 & 53,21 \\
\hline \multicolumn{5}{|l|}{ Prazos Médios (dias) } \\
\hline Prazo Médio de Estoques - PME & 101,09 & 50,49 & 4,78 & 80,43 \\
\hline Prazo Médio de Recebimentos - PMR & 89,12 & 50,84 & 12,07 & 57,68 \\
\hline Prazo Médio de Pagamentos - PMP & 14,58 & 41,23 & 90,48 & 60,95 \\
\hline Ciclo Operacional - CO & 186,56 & 104,10 & 25,10 & 138,11 \\
\hline Ciclo Financeiro - CF & 104,54 & 61,78 & 0,59 & 77,02 \\
\hline \multicolumn{5}{|l|}{ Capital de Giro (\%) } \\
\hline Capital Circulante Líquido - CCL & 11,34 & 18,82 & 33,80 & 22,97 \\
\hline Necessidade de Capital de Giro - NCG & 33,86 & 7,48 & $-2,99$ & 11,61 \\
\hline Saldo de Tesouraria - ST & $-5,77$ & 6,92 & 18,44 & 10,20 \\
\hline
\end{tabular}

Fonte: Os autores (2019).

Tendo por base os quartis obtidos para cada indicador, foi possível avaliar os índices de cada cooperativa e classificá-los, conforme o desempenho, em excelentes, bons, regulares e insatisfatórios. 


\subsection{1 Índice de Solvência}

Na tabela 3 são apresentados os índices LC, LS e LG, classificados de acordo com os índicespadrão encontrados.

Tabela 3 - Índices de Solvência

\begin{tabular}{|c|c|c|c|c|c|c|c|c|c|c|c|c|c|c|c|c|c|}
\hline \multicolumn{18}{|c|}{ Liquidez Corrente } \\
\hline \multicolumn{4}{|c|}{ INSATISFATÓRIO } & \multicolumn{5}{|c|}{ REGULAR } & \multicolumn{5}{|c|}{ BOM } & \multicolumn{4}{|c|}{ EXCELENTE } \\
\hline 0,33 & 0,90 & 1,03 & 1,10 & 1,14 & 1,22 & 1,45 & 1,46 & 1,49 & 1,57 & 1,66 & 1,87 & 1,92 & 1,93 & 2,67 & 3,06 & 3,75 & 8,49 \\
\hline I & $\mathbf{A}$ & B & $\mathbf{R}$ & $\mathbf{L}$ & D & $\mathbf{P}$ & $\mathbf{J}$ & C & $\mathbf{0}$ & $\mathbf{N}$ & $\mathbf{Q}$ & G & $\mathbf{K}$ & $\mathbf{M}$ & $\mathbf{F}$ & $\mathbf{E}$ & $\mathbf{H}$ \\
\hline \multicolumn{18}{|c|}{ Liquidez Seca } \\
\hline \multicolumn{4}{|c|}{ INSATISFATÓRIO } & \multicolumn{5}{|c|}{ REGULAR } & \multicolumn{5}{|c|}{ BOM } & \multicolumn{4}{|c|}{ EXCELENTE } \\
\hline 0,33 & 0,49 & 0,70 & 0,70 & 0,91 & 0,99 & 1,05 & 1,10 & 1,10 & 1,19 & 1,30 & 1,35 & 1,40 & 1,40 & 1,85 & 2,14 & 2,33 & 8,49 \\
\hline I & B & A & D & $\mathbf{L}$ & $\mathbf{J}$ & G & $\mathbf{R}$ & C & $\mathbf{P}$ & $\mathbf{0}$ & $\mathbf{K}$ & $\mathbf{M}$ & $\mathbf{N}$ & $\mathbf{Q}$ & $\mathbf{F}$ & $\mathbf{E}$ & $\mathbf{H}$ \\
\hline \multicolumn{18}{|c|}{ Liquidez Geral } \\
\hline \multicolumn{4}{|c|}{ INSATISFATÓRIO } & \multicolumn{5}{|c|}{ REGULAR } & \multicolumn{5}{|c|}{ BOM } & \multicolumn{4}{|c|}{ EXCELENTE } \\
\hline 0,33 & 0,53 & 1,02 & 1,08 & 1,08 & 1,08 & 1,10 & 1,14 & 1,16 & 1,21 & 1,38 & 1,54 & 1,56 & 1,67 & 2,94 & 3,75 & 3,85 & 8,49 \\
\hline I & $\mathbf{A}$ & B & 0 & D & K & $\mathbf{R}$ & $\mathbf{L}$ & $\mathbf{J}$ & $\mathbf{P}$ & $\mathbf{N}$ & F & C & $\mathbf{Q}$ & G & $\mathbf{E}$ & $\mathbf{M}$ & $\mathbf{H}$ \\
\hline
\end{tabular}

Fonte: Os autores (2019).

Os índices de LC apresentaram o melhor cenário do grupo de solvência. Do total da amostra, dezesseis cooperativas possuem um ativo circulante maior que o passivo circulante, o que possibilita cumprirem com suas obrigações em curto prazo.

Com relação a LS, seis delas apresentaram valores abaixo de 1 e a mediana, em confronto com os valores de LC, apresentados na Tabela 1, caiu 38 centavos. 0 cenário para análise da LG também revela menor mediana que a corrente, com diferença de 35 centavos, porém apenas duas das dezoito cooperativas apresentam valores abaixo de 1 .

A Cooperativa $\mathrm{H}$ apresentou o mesmo resultado do índice de liquidez corrente, seca e geral, pois não apresenta estoques, realizável a longo prazo, nem exigível de longo prazo.

\subsection{2 Índice de Rentabilidade}

A análise dos índices de rentabilidade permite mensurar o quanto a entidade pode se apresentar rentável ou não, através de capitais investidos. 
Tabela 4 - Índices de Rentabilidade

\begin{tabular}{|c|c|c|c|c|c|c|c|c|c|c|c|c|c|c|c|c|c|}
\hline \multicolumn{18}{|c|}{ Rentabilidade do Ativo (\%) } \\
\hline \multicolumn{4}{|c|}{ INSATISFATÓRIO } & \multicolumn{5}{|c|}{ REGULAR } & \multicolumn{5}{|c|}{ BOM } & \multicolumn{4}{|c|}{ EXCELENTE } \\
\hline$-9,84$ & $-8,90$ & $-1,29$ & $-1,24$ & $-0,59$ & 0,09 & 0,26 & 0,45 & 1,38 & 1,61 & 2,20 & 2,60 & 3,84 & 6,65 & 6,86 & 7,68 & 9,39 & 17,42 \\
\hline $\mathbf{E}$ & G & C & $\mathbf{R}$ & $\mathbf{D}$ & $\mathbf{P}$ & $\mathbf{Q}$ & B & $\mathbf{F}$ & $\mathbf{N}$ & $\mathbf{J}$ & $\mathbf{0}$ & $\mathbf{L}$ & $\mathbf{M}$ & $\mathbf{A}$ & I & $\mathbf{K}$ & $\mathbf{H}$ \\
\hline \multicolumn{18}{|c|}{ Rentabilidade do Patrimônio Líquido (\%) } \\
\hline \multicolumn{4}{|c|}{ INSATISFATÓRIO } & \multicolumn{5}{|c|}{ REGULAR } & \multicolumn{5}{|c|}{ BOM } & \multicolumn{4}{|c|}{ EXCELENTE } \\
\hline$-13,48$ & $-11,65$ & $-10,93$ & $-3,71$ & $-2,33$ & 0,18 & 0,56 & 1,70 & 3,26 & 4,08 & 5,52 & 7,58 & 8,70 & 9,12 & 16,02 & 19,65 & 23,25 & 29,05 \\
\hline $\mathbf{R}$ & $\mathbf{E}$ & G & D & C & $\mathbf{P}$ & $\mathbf{Q}$ & $\mathbf{F}$ & $\mathbf{N}$ & $\mathbf{J}$ & $\mathbf{0}$ & B & $\mathbf{M}$ & I & A & H & $\mathbf{K}$ & $\mathbf{L}$ \\
\hline \multicolumn{18}{|c|}{ Giro do Ativo (dias) } \\
\hline \multicolumn{4}{|c|}{ INSATISFATÓRIO } & \multicolumn{5}{|c|}{ REGULAR } & \multicolumn{5}{|c|}{ BOM } & \multicolumn{4}{|c|}{ EXCELENTE } \\
\hline 0,28 & 0,70 & 0,79 & 0,85 & 1,06 & 1,17 & 1,17 & 1,29 & 1,39 & 1,53 & 1,94 & 2,77 & 2,82 & 3,09 & 4,04 & 4,37 & 5,91 & 14,38 \\
\hline $\mathbf{F}$ & B & D & $\mathbf{L}$ & $\mathbf{E}$ & $\mathbf{0}$ & $\mathbf{J}$ & G & $\mathbf{H}$ & $\mathbf{M}$ & $\mathbf{K}$ & I & A & $\mathbf{P}$ & $\mathbf{N}$ & C & $\mathbf{R}$ & $\mathbf{Q}$ \\
\hline \multicolumn{18}{|c|}{ Margem Líquida (\%) } \\
\hline \multicolumn{4}{|c|}{ INSATISFATÓRIO } & \multicolumn{5}{|c|}{ REGULAR } & \multicolumn{5}{|c|}{ BOM } & \multicolumn{4}{|c|}{ EXCELENTE } \\
\hline $\begin{array}{l}-9,33 \\
\end{array}$ & $-6,90$ & $-0,75$ & $-0,21$ & 0,00 & 0,02 & 0,03 & 0,40 & 0,64 & 1,87 & 2,23 & 2,43 & 2,77 & 4,36 & 4,49 & 4,85 & 5,01 & 17,73 \\
\hline E & G & D & $\mathbf{R}$ & C & $\mathbf{Q}$ & $\mathbf{P}$ & $\mathbf{N}$ & B & $\mathbf{J}$ & 0 & A & I & $\mathbf{M}$ & $\mathbf{L}$ & $\mathbf{K}$ & $\mathbf{F}$ & H \\
\hline
\end{tabular}

Fonte: Os autores (2019).

A ROA evidenciou que cinco das dezoito cooperativas apresentaram resultados negativos. As demais figuram com retornos positivos, porém, o maior valor auferido como lucro pelas cooperativas objeto da amostra é $R \$ 17,42$, para cada $R \$ 100,00$ de investimento.

Enfatiza-se que, a ROA cresce ou diminui à medida que o GA e a ML apresentam melhor ou pior configuração, ou até mesmo pela compensação que um traz ao outro. Como exemplo, pode-se observar a cooperativa E, que apresentou conceito "insatisfatório" para a margem líquida, e "regular" para o GA, e consequentemente, foi avaliada com conceito "insatisfatório" para a rentabilidade do ativo. Já a cooperativa $H$, apresentou conceito "excelente" para a rentabilidade do ativo, pois apesar de apresentar o GA menor que o padrão, obteve conceito "excelente" na ML.

\subsection{3 Índices de Estrutura de Capital}

Os índices de estrutura de capital revelam as linhas de decisões financeiras quanto às captações de recursos e suas aplicações.

Tabela 5 - Índices de Estrutura de Capital (\%)

\begin{tabular}{|c|c|c|c|c|c|c|c|c|c|c|c|c|c|c|c|c|c|}
\hline \multicolumn{18}{|c|}{ Participação de Capital de Terceiros } \\
\hline \multicolumn{4}{|c|}{ INSATISFATÓRIO } & \multicolumn{5}{|c|}{ REGULAR } & \multicolumn{5}{|c|}{ BOM } & \multicolumn{4}{|c|}{ EXCELENTE } \\
\hline 1597,7 & 991,6 & 657,4 & 526,1 & 147,7 & 133,4 & 113,5 & 112,0 & 102,6 & 93,4 & 85,8 & 80,5 & 30,8 & 23,2 & 22,8 & 18,7 & 18,4 & 12,8 \\
\hline B & $\mathbf{R}$ & $\mathbf{L}$ & D & $\mathbf{K}$ & $\mathbf{A}$ & $\mathbf{Q}$ & $\mathbf{0}$ & $\mathbf{N}$ & $\mathbf{P}$ & $\mathbf{J}$ & C & M & $\mathbf{F}$ & G & I & $\mathbf{E}$ & $\mathbf{H}$ \\
\hline \multicolumn{18}{|c|}{ Composição do Endividamento } \\
\hline \multicolumn{4}{|c|}{ INSATISFATÓRIO } & \multicolumn{5}{|c|}{ REGULAR } & \multicolumn{5}{|c|}{ BOM } & \multicolumn{4}{|c|}{ EXCELENTE } \\
\hline 100,0 & 100,0 & 100,0 & 100,0 & 99,8 & 99,8 & 95,0 & 83,1 & 82,8 & 81,7 & 78,9 & 77,8 & 75,5 & 71,1 & 64,5 & 56,1 & 54,8 & 50,3 \\
\hline $\mathbf{E}$ & $\mathbf{H}$ & I & $\mathbf{M}$ & $\mathbf{L}$ & $\mathbf{R}$ & C & $\mathbf{N}$ & $\mathbf{P}$ & D & $\mathbf{Q}$ & B & G & $\mathbf{J}$ & $\mathbf{0}$ & $\mathbf{K}$ & $\mathbf{A}$ & $\mathbf{F}$ \\
\hline \multicolumn{18}{|c|}{ Imobilizado do Patrimônio Líquido } \\
\hline \multicolumn{4}{|c|}{ INSATISFATÓRIO } & \multicolumn{5}{|c|}{ REGULAR } & \multicolumn{5}{|c|}{ BOM } & \multicolumn{4}{|c|}{ EXCELENTE } \\
\hline 112,4 & 94,1 & 92,1 & 87,9 & 85,9 & 80,6 & 79,7 & 60,7 & 58,3 & 55,7 & 53,7 & 48,0 & 22,8 & 12,2 & 5,9 & 2,7 & 2,5 & 2,4 \\
\hline I & A & 0 & $\mathbf{K}$ & $\mathbf{J}$ & $\mathbf{F}$ & $\mathbf{P}$ & $\mathbf{N}$ & D & G & C & $\mathbf{E}$ & $\mathbf{Q}$ & $\mathbf{M}$ & $\mathbf{R}$ & H & B & $\mathbf{L}$ \\
\hline
\end{tabular}


O PCT revelou que todas as empresas com desempenho "insatisfatório" e "regular", para cada $R \$ 100,00$ de capital próprio investido, a cooperativa tomou pelo menos $R \$ 102,63$ de capital de terceiros, evidenciando uma alta dependência de capitais de terceiros.

0 índice de CE revelou que todas as entidades com conceito "insatisfatório", possuem suas obrigações totalmente dependentes de dívidas de curto prazo. Destaca-se que as cooperativas enquadradas com conceito "bom", ainda assim possuem mais de $70 \%$ de suas obrigações a curto prazo.

Quanto ao IPL, infere-se que a cooperativa I, além de ter investido todo seu capital próprio em ativos de investimento, imobilizado e intangível, ela usou $R \$ 12,36$ originado de capital de terceiros. Diante desse exemplo, pode-se concluir que quanto mais as cooperativas investem em ativos permanentes, mais elas dependem de capitais de terceiros para financiar o Ativo Circulante.

\subsection{4 Índices de Prazos Médios}

Os índices de PME, PMR e PMP compõem o CO e CF das cooperativas e interagem, entre outros índices, como a NCG.

Tabela 6 - Índices de Prazos Médios (dias)

\begin{tabular}{|c|c|c|c|c|c|c|c|c|c|c|c|c|c|c|c|c|}
\hline \multicolumn{17}{|c|}{ Prazo Médio de Estoques } \\
\hline \multicolumn{4}{|c|}{ INSATISFATÓRIO } & \multicolumn{4}{|c|}{ REGULAR } & \multicolumn{5}{|c|}{ BOM } & \multicolumn{4}{|c|}{ EXCELENTE } \\
\hline 563,9 & 216,2 & 109,7 & 107,3 & 94,9 & 85,2 & 78,3 & 55,7 & 50,5 & 34,3 & 16,7 & 13,3 & 12,7 & 9,3 & 0,3 & 0,0 & 0,0 \\
\hline D & B & $\mathbf{F}$ & $\mathbf{M}$ & $\mathbf{L}$ & $\mathbf{K}$ & $\mathbf{E}$ & $\mathbf{J}$ & G & $\mathbf{0}$ & C & $\mathbf{N}$ & $\mathbf{P}$ & $\mathbf{A}$ & $\mathbf{Q}$ & $\mathbf{I}$ & $\mathbf{R}$ \\
\hline \multicolumn{17}{|c|}{ Prazo Médio de Recebimento } \\
\hline \multicolumn{4}{|c|}{ INSATISFATÓRIO } & \multicolumn{4}{|c|}{ REGULAR } & \multicolumn{5}{|c|}{ BOM } & \multicolumn{4}{|c|}{ EXCELENTE } \\
\hline 178,1 & 144,9 & 107,3 & 93,3 & 85,0 & 69,8 & 65,9 & 64,7 & 50,8 & 47,8 & 39,1 & 31,1 & 24,1 & 12,9 & 11,3 & 9,6 & 2,7 \\
\hline $\mathbf{L}$ & B & D & $\mathbf{K}$ & $\mathbf{F}$ & $\mathbf{0}$ & $\mathbf{E}$ & $\mathbf{M}$ & $\mathbf{J}$ & $\mathbf{R}$ & $\mathbf{N}$ & G & $\mathbf{P}$ & $\mathbf{A}$ & C & $\mathbf{Q}$ & I \\
\hline \multicolumn{17}{|c|}{ Prazo Médio de Pagamento } \\
\hline \multicolumn{4}{|c|}{ INSATISFATÓRIO } & \multicolumn{4}{|c|}{ REGULAR } & \multicolumn{5}{|c|}{ BOM } & \multicolumn{4}{|c|}{ EXCELENTE } \\
\hline 0,0 & 4,5 & 11,6 & 17,6 & 19,8 & 23,2 & 23,7 & 34,8 & 41,2 & 42,3 & 46,7 & 64,7 & 80,2 & 100,8 & 147,3 & 199,9 & 238,7 \\
\hline $\mathbf{N}$ & I & $\mathbf{Q}$ & $\mathbf{0}$ & G & $\mathbf{A}$ & C & $\mathbf{P}$ & $\mathbf{F}$ & $\mathbf{E}$ & $\mathbf{R}$ & $\mathbf{M}$ & $\mathbf{K}$ & $\mathbf{J}$ & B & $\mathbf{L}$ & D \\
\hline \multicolumn{17}{|c|}{ Ciclo Operacional } \\
\hline \multicolumn{4}{|c|}{ INSATISFATÓRIO } & \multicolumn{4}{|c|}{ REGULAR } & \multicolumn{5}{|c|}{ BOM } & \multicolumn{4}{|c|}{ EXCELENTE } \\
\hline 671,2 & 361,1 & 273,1 & 194,7 & 178,5 & 171,9 & 144,2 & 106,6 & 104,1 & 81,6 & 52,4 & 47,8 & 36,7 & 28,0 & 22,2 & 9,3 & 2,7 \\
\hline D & B & $\mathbf{L}$ & $\mathbf{F}$ & $\mathbf{K}$ & $\mathbf{M}$ & $\mathbf{E}$ & $\mathbf{J}$ & $\mathbf{0}$ & G & $\mathbf{N}$ & $\mathbf{R}$ & $\mathbf{P}$ & C & $\mathbf{A}$ & $\mathbf{Q}$ & I \\
\hline \multicolumn{17}{|c|}{ Ciclo Financeiro } \\
\hline \multicolumn{4}{|c|}{ INSATISFATÓRIO } & \multicolumn{4}{|c|}{ REGULAR } & \multicolumn{5}{|c|}{ BOM } & \multicolumn{4}{|c|}{ EXCELENTE } \\
\hline 432,5 & 213,8 & 153,4 & 107,2 & 101,9 & 98,3 & 86,5 & 73,2 & 61,8 & 52,4 & 5,8 & 4,3 & 1,9 & 1,2 & $-1,0$ & $-2,3$ & $-4,5$ \\
\hline D & B & $\mathbf{F}$ & $\mathbf{M}$ & $\mathbf{E}$ & $\mathbf{K}$ & $\mathbf{0}$ & $\mathbf{L}$ & G & $\mathbf{N}$ & $\mathbf{J}$ & C & $\mathbf{P}$ & $\mathbf{R}$ & $\mathbf{A}$ & $\mathbf{Q}$ & I \\
\hline
\end{tabular}

Fonte: Os autores (2019).

As cooperativas que apresentam PME de menos de quinze dias foram classificadas, de acordo com os índice-padrão da amostra, como "excelentes" e uma como "bom". Porém, partir de cinquenta e um dias, essas passaram a ter qualificação de "regular" e "insatisfatório". 0 PMR por sua vez, classificou como "excelente" de acordo com a amostra, cooperativas com recebimentos após as vendas de pelo menos de vinte e cinco dias. 0 indicador de PMP classificou como "excelente" os prazos de pagamento a terceiros com pelo menos 80 dias. 0 CO para ser considerado "excelente" nesta amostra, deve apresentar menos de dois dias de financiamento do ciclo operacional com capital próprio. 
As cooperativas I e R não possuem estoque, e por isso apresentam PME igual a zero. Já a cooperativa $\mathrm{N}$ não apresenta saldo nas contas de fornecedor e cooperados a pagar, motivo pelo qual o PMP mostra-se zerado. Cabe destacar que a cooperativa $\mathrm{H}$ foi excluída da classificação dos índices de prazos médios, porquanto não apresenta valores suficientes para o cálculo de nenhum indicador desse grupo.

\subsubsection{Dinâmica de Capital de Giro}

A análise dos ativos e passivos correntes garante equilíbrio e estabilidade financeira para a organização e exerce influência sobre índices de liquidez e rentabilidade.

Tabela 7 - Dinâmica de Capital de Giro (\%)

\begin{tabular}{|c|c|c|c|c|c|c|c|c|c|c|c|c|c|c|c|c|c|}
\hline \multicolumn{18}{|c|}{ Capital Circulante Líquido } \\
\hline \multicolumn{4}{|c|}{ INSATISFATÓRIO } & \multicolumn{5}{|c|}{ REGULAR } & \multicolumn{5}{|c|}{ BOM } & \multicolumn{4}{|c|}{ EXCELENTE } \\
\hline$-3,2$ & 2,2 & 8,8 & 10,5 & 12,2 & 12,9 & 14,9 & 15,1 & 18,2 & 19,4 & 19,5 & 20,9 & 27,7 & 30,9 & 36,7 & 39,2 & 42,8 & 84,9 \\
\hline $\mathbf{A}$ & B & $\mathbf{R}$ & I & $\mathbf{L}$ & G & D & $\mathbf{J}$ & $\mathbf{P}$ & $\mathbf{0}$ & $\mathbf{F}$ & C & $\mathbf{N}$ & $\mathbf{K}$ & $\mathbf{Q}$ & $\mathbf{M}$ & $\mathbf{E}$ & $\mathbf{H}$ \\
\hline \multicolumn{18}{|c|}{ Necessidade de Capacidade de Giro } \\
\hline \multicolumn{4}{|c|}{ INSATISFATÓRIO } & \multicolumn{5}{|c|}{ REGULAR } & \multicolumn{5}{|c|}{ BOM } & \multicolumn{4}{|c|}{ EXCELENTE } \\
\hline 47,1 & 39,1 & 38,2 & 33,9 & 33,9 & 27,5 & 12,2 & 9,9 & 8,3 & 6,6 & 1,4 & $-1,0$ & $-1,9$ & $-2,6$ & $-3,4$ & $-4,6$ & $-11,3$ & $-24,2$ \\
\hline $\mathbf{N}$ & $\mathbf{B}$ & $\mathbf{0}$ & $\mathbf{K}$ & $\mathbf{M}$ & $\mathbf{E}$ & $\mathbf{F}$ & G & D & C & $\mathbf{J}$ & $\mathbf{R}$ & I & $\mathbf{Q}$ & $\mathbf{P}$ & $\mathbf{A}$ & $\mathbf{H}$ & $\mathbf{L}$ \\
\hline \multicolumn{18}{|c|}{ Saldo em Tesouraria } \\
\hline \multicolumn{4}{|c|}{ INSATISFATÓRIO } & \multicolumn{5}{|c|}{ REGULAR } & \multicolumn{5}{|c|}{ BOM } & \multicolumn{4}{|c|}{ EXCELENTE } \\
\hline$-36,9$ & $-19,4$ & $-18,6$ & $-8,6$ & $-2,9$ & 1,4 & 3,1 & 5,3 & 6,6 & 7,3 & 9,8 & 13,7 & 14,3 & 15,3 & 21,6 & 36,3 & 39,2 & 96,3 \\
\hline B & $\mathbf{N}$ & $\mathbf{0}$ & I & $\mathbf{K}$ & $\mathbf{A}$ & G & M & D & $\mathbf{F}$ & $\mathbf{R}$ & J & C & $\mathbf{E}$ & $\mathbf{P}$ & $\mathbf{L}$ & $\mathbf{Q}$ & $\mathbf{H}$ \\
\hline
\end{tabular}

Fonte: Os autores (2019).

Em relação ao CCL, a cooperativa $\mathrm{H}$ destacou-se dentro da amostra calculada, pois além de apresentar o maior CCL, também possui seu índice bem acima do índice padrão. A cooperativa A, em contrapartida, apresentou desempenho negativo, o que representa que suas dívidas de curto prazo são superiores aos ativos de curto prazo.

Todas as cooperativas com NCG negativa, apresentaram conceito "excelente" ou "bom", representando uma sobra de recurso das atividades operacionais, ressaltando que saber administrar operações de curto prazo e obter retornos rápidos influenciam não somente no NCG, mas também causam impacto no ciclo operacional.

\subsection{Análise de Desempenho das Cooperativas}

A figura 1 apresenta a frequência dos conceitos - insatisfatório, regular, bom e excelente atribuídos aos índices econômico-financeiros, por cooperativa, no qual se pode observar o desempenho geral das cooperativas da amostra. Os percentuais correspondem à quantidade em que os indicadores foram classificados em cada categoria. 
Figura 1 - Frequência de cada conceito por cooperativa

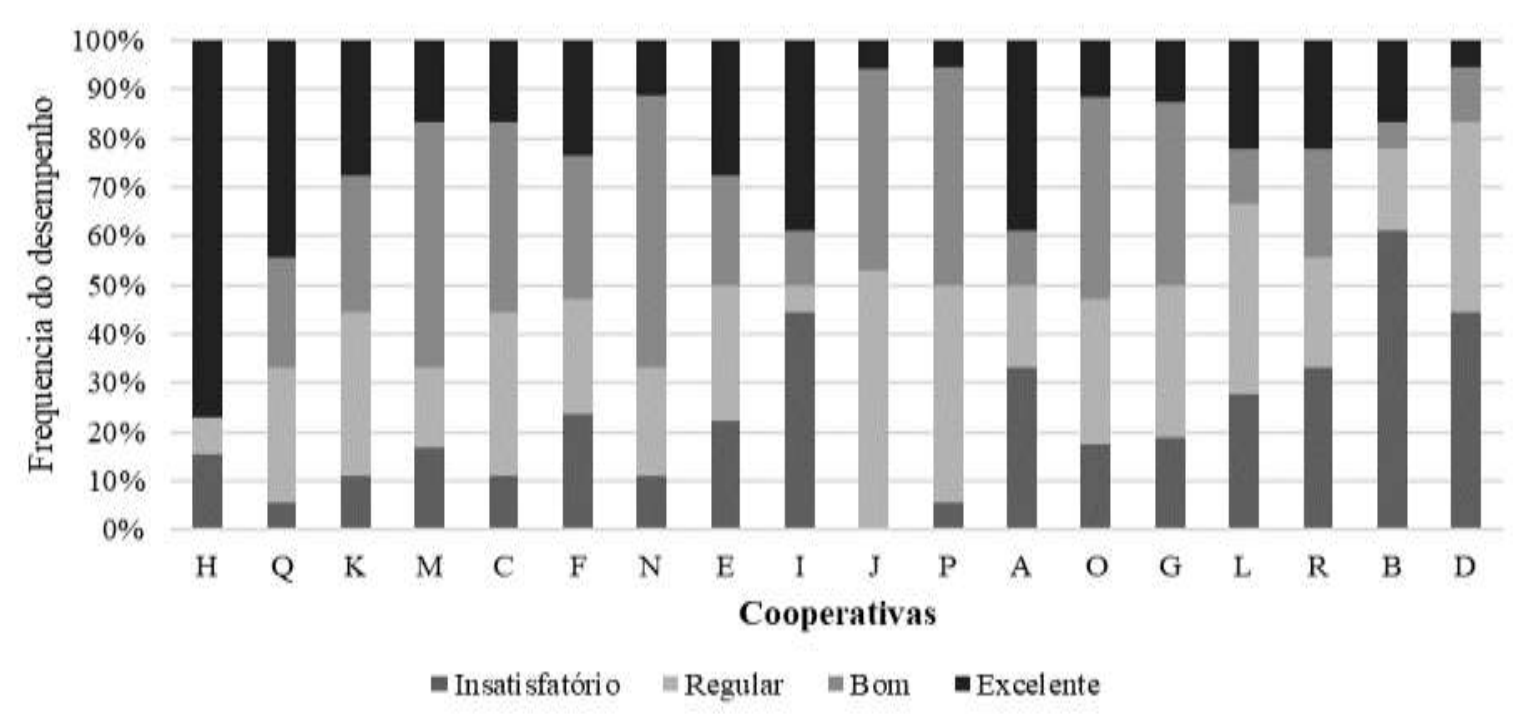

Fonte: Os autores (2019).

Nota-se que as cooperativas $\mathrm{P}$ e J não apresentaram nenhum índice cujo conceito fosse "insatisfatório", enquanto a B, apresentou $65 \%$ de seus indicadores como "insatisfatórios" e $20 \%$ como "regulares", totalizando $85 \%$ de seus índices abaixo do padrão. Já a cooperativa $\mathrm{H}$, apresentou maior frequência de índices classificados como "excelentes", e apenas 13\% abaixo do padrão setorial.

\section{Considerações Finais}

O escopo deste trabalho foi desenvolver, por meio de índices-padrão, um referencial de comparação para os índices econômico-financeiros das cooperativas agropecuárias do Estado de Mato Grosso do Sul.

Fez-se a escolha por cooperativas do ramo agropecuário devido à relevância da atividade para o Estado e para o País e pelas perspectivas de crescimento do agronegócio.

A partir do que foi desenvolvido, será possível que as cooperativas do Mato Grosso do Sul, ao realizarem a análise de seus demonstrativos financeiros, comparem seu desempenho com a média setorial, evidenciando a importância deste estudo, já que, por se tratar de um ambiente competitivo, exige-se o bom desempenho das empresas perante o mercado, além da gestão eficiente de seus recursos. Os resultados também podem ser úteis para o Sistema OCB/MS no sentido de acompanhar o desempenho econômico-financeiro das suas afiliadas, com base nos indicadores-padrão construídos.

Estudos mais aprofundados poderão analisar o comportamento individual de cada cooperativa em relação ao padrão setorial. Sugere-se, portanto, para pesquisas futuras a avaliação não apenas de indicadores financeiros, mas também a correlação destes com os não financeiros, visto que o sistema cooperativo almeja não só ter um bom desempenho, mas principalmente fornecer produtos e serviços de qualidade, atendendo os objetivos dos cooperados e promovendo o desenvolvimento social.

Sugere-se também a comparação dos indicadores-padrão construídos para as cooperativas do Mato Grosso do Sul com cooperativas de outros estados, visando uma análise comparativa de abrangência nacional.

\section{Referências}

ALMEIDA, L. B. et al. A Utilização do orçamento como ferramenta de apoio à formulação de estratégia, de controle e de interatividade: um estudo exploratório nas cooperativas 
agropecuárias da região sul do Brasil. Contabilidade Vista \& Revista, v. 20, n. 3, p. 65-99, jul./set. 2009.

ASSAF NETO, A. Estrutura e análise de balanços: um enfoque econômico-financeiro. 11. ed. São Paulo: Atlas, 2015.

ARANHA, J. A. M. Análise financeira e índices padrão para o setor elétrico brasileiro. 2015. $211 \mathrm{f}$. Dissertação (Mestrado em Ciências Contábeis e Financeira) - Pontifícia Universidade Católica de São Paulo, São Paulo, 2015.

BENTO, M. H. S.; OLIVEIRA, E.; MADRUGA, L. R. R.G. Análise Econômica-Financeira em Cooperativas: um estudo de caso na CAMSUL. Revista de Gestão e Organizações Cooperativas, Santa Maria, v. 3, n. 6, p. 15-30, 2016.

BIALOSKORSKI NETO, S. Um ensaio sobre o desempenho econômico e participação em cooperativas agropecuárias. Revista de Economia e Sociologia Rural, Brasília, v. 45, no 1, Jan-Mar. 2007.

BRASIL. Lei no 5.764, de 16 de dezembro de 1971. Define a Política Nacional de Cooperativismo, institui o regime jurídico das sociedades cooperativas, e dá outras providências. Disponível em: http://www.planalto.gov.br/ccivil_03/LEIS/L5764.HTM. Acesso em: 04 abr. 2019.

BRASIL. Ministério da Indústria, Comércio Exterior e Serviços. Balança Comercial Brasileira: Cooperativas. Disponível em: http://www.mdic.gov.br/index.php/comercio-exterior/estatisticas-decomercio-exterior/balanca-comercial-brasileira-cooperativas. Acesso em: 18 out. 2017.

CARPES, A. M.S.; ORO, I. M. Análise do ambiente de controle interno nas cooperativas agropecuárias do estado de Santa Catarina: uma investigação alicerçada na perspectiva da metodologia COSO (the Comitee of Sposoring Organization). In: 7o CONGRESSO USP DE INICIAÇÃO CIENTÍFICA EM CONTABILIDADE. 2010, São Paulo. Anais. São Paulo: USP, 2010.

CARPES, A. M.S.; SCHONS, P.; OLIVEIRA, L. Nível de disclosure praticado pelas cooperativas do estado de Santa Catarina no tocante às orientações emanadas pela NBCT 10.8. In: CONTECSIInternational Conference on Information Systems and Technology Management. São Paulo. Anais. São Paulo: 2012.v. 9, p. 480-498.

CARVALHO, F. L. et al. Saída e insucesso das cooperativas de crédito no Brasil: uma análise do risco. Revista Contabilidade \& Finanças-USP, São Paulo, v. 26, n. 67, p. 70-84, jan./fev./mar./abr. 2015.

CORRÊA, R. G. F.; KLIEMANN NETO, F. J.; DENICOL, J. o agronegócio sob a ótica da gestão econômico-financeira: uma revisão. Agroalimentaria, v. 23, n. 44, p. 61-77, jan./jun., 2017.

DIEL, F. J. et al. Análise da eficiência econômico-financeira das empresas pertencentes ao agronegócio brasileiro. CONTEXTUS Revista Contemporânea de Economia e Gestão, Fortaleza, v. 12, n. 2, p. 116-133, mai./ago. 2014.

DUARTE, H. C. F.; LAMOUNIER, W. M. Análise Financeira de Empresas da Construção Civil por Comparação com Índices-Padrão. Revista Enfoque Reflexão Contábil, v. 26, n. 2, p. 09-28, maio/ago., 2007.

GIL, A. C. Métodos e técnicas de pesquisa social. 6. ed. São Paulo: Atlas, 2008.

GIMENES, R. M. T. Novos padrões de financiamento para as cooperativas agropecuárias. $146 \mathrm{f}$. Relatório de Pesquisa (Pós-doutorado em Administração de Empresas) - Programa de Pós-doutorado em Administração de Empresas da FEA/USP, São Paulo, 2006. 
GIMENES, R. M. T.; GIMENES, F. M. P. Aplicabilidade da análise dinâmica do capital de giro como instrumento de avaliação da gestão financeira em cooperativas agropecuárias. Revista de Economia Contemporânea, Rio de Janeiro, v. 12, n. 1, p. 129-150, jan/abr. 2008.

GIMENES, R. M. T.; GIMENES, F. M. P. Desafios para a gestão financeira das cooperativas agropecuárias brasileiras. Análise, Porto Alegre, v. 19, n. 1, p. 76-98, jan./jun. 2008.

GOZER, I. C. et al. Avaliação econômico-financeira de cooperativas agropecuárias utilizando índices-padrão do setor: um estudo de caso. In: XLV Congresso da Sociedade Brasileira de Economia, Administração e Sociologia Rural, 2007.

INTERNATIONAL CO-OPERATIVE ALLIANCE. Base de dados. 2017. Disponível em: https://ica.coop/en/what-co-operative. Acesso em: 18 out. 2017.

INSTITUTO BRASILEIRO DE GEOGRAFIA E ESTATÍSTICA (IBGE). 2017. Contas Nacionais e Trimestrais: Indicadores de Volume e Valores Correntes. Disponível em: https://biblioteca.ibge.gov.br/visualizacao/periodicos/2121/cnt_2017_1tri.pdf. Acesso em: 19 maio 2017.

INSTITUTO BRASILEIRO DE GEOGRAFIA E ESTATÍSTICA (IBGE).PIB cresce 1,1\% em 2018 e fecha ano em R\$ 6,8 trilhões. Disponível em: https://agenciadenoticias.ibge.gov.br/agencia-sala-deimprensa/2013-agencia-de-noticias/releases/23886-pib-cresce-1-1-em-2018-e-fecha-ano-em-r-6-8trilhoes. Acesso em: 07 jun. 2019.

JAHARA, R. C.; MELlO, J. A. V. B.; AFONSO, H. C. A. G. Proposta de Índice Padrão e Análise de Performance Financeira dos Clubes Brasileiros de Futebol da Série A no Ano 2014. Podium Sport, Leisure and Tourism Review, v. 5, n. 3, p. 20-40, set./dez. 2016.

JANK, M. S.; NASSAR, A. M.; TACHINARDI, M. H. Agronegócio e Comércio Exterior Brasileiro. Revista USP, São Paulo, n. 64, p. 14-27, dez/fev. 2004-2005.

KALOGERAS, N. et al. Which Cooperative Ownership Model Performs Better? A FinancialDecision Aid Approach. Agribusiness, v. 29, n. 1, p. 80-95, 2013.

LAUERMANN, G. J. et al. Desempenho econômico-financeiro de cooperativas: o caso do programa de monitoramento da autogestão das cooperativas agropecuárias do Paraná. Revista de Gestão e Organizações Cooperativas, Santa Maria, v. 3, n. 6, p. 59-72, 2016.

LOPES, A. C. V.; MENEZES, E. A. Gestão financeira das cooperativas: aplicação do modelo dinâmico. Revista Gestão Industrial, Ponta Grossa, v. 2, n. 2, p. 136 - 145, 2006.

MATARAZZO, D. C. Análise financeira de balanços: abordagem gerencial. 7. ed. São Paulo: Atlas, 2010.

MINISTÉRIO DA AGRICULTURA, PECUÁRIA E ABASTECIMENTO (MAPA). Participação do agronegócio nas exportações brasileiras cresce $1,5 \%$ em março. Disponível em: http://www.agricultura.gov.br/noticias/participacao-do-agronegocio-nas-exportacoes-brasileirascresce-1-5-em-marco. Acesso em: 07 jun. 2019.

MOREIRA, V. R.; BARREIROS, R. F.; PROTIL, R. M. Portfolio de produção agropecuária e gestão de riscos de mercado nas cooperativas do agronegócio paranaense. Revista de Administração, São Paulo, v. 46, n. 4, p. 325-341, 2011.

MUNARETTO, L. F.; CORREAA, H. L. Indicadores de Desempenho Organizacional: Uso e Finalidades nas Cooperativas de Eletrificação do Brasil. Revista Contabilidade Vista e Revista, Belo Horizonte, v. 27, n. 1, p. 25-41, jan/abr. 2016. 
ORGANIZAÇÃO DAS COOPERATIVAS BRASILEIRAS. Base de dados. Disponível em: http://www.ocb.org.br/. Acesso em: 13 jun. 2017.

ORGANIZAÇÃO DAS COOPERATIVAS BRASILEIRAS DO MATO GROSSO DO SUL. Relatório de Gestão, $2016 . \quad$ Disponível em: http://www.ocb.org.br/arquivos/RelatorioAnual/relatorio_de_gestao_ocb_2016.pdf. Acesso em: 13 jun. 2017.

ORGANIZAÇÃO INTERNACIONAL DO TRABALHO, Recomendação 193 de 2002. Disponível em: http://www.oitbrasil.org.br/content/sobre-promo\%C3\%A7\%C3\%A3o-de-cooperativas. Acesso em: jun. 2017.

PEREIRA, B. A. D. et al. Análise da eficiência em cooperativas agropecuárias no Estado do Rio Grande do Sul. Revista Universo Contábil, FURB, Blumenau, v. 5, n. 2, p. 39-57, abr./jun. 2009.

PEREIRA, G.; CARVAlHO, F. N.; PARENTE, E. G. V. Desempenho econômico e evidenciação ambiental: análise das empresas que receberam o Prêmio Rumo à Credibilidade 2010. Revista Catarinense da Ciência Contábil, v. 10, n. 30, p. 09-26, ago./nov. 2011.

RENDÓN-ÁLVAREZ, B.; RODRÍGUEZ-BOLAÑOS, J.; RIASCOS-GÓMEZ, P. A. Análisis del impacto en el capital institucional de las cooperativas de ahorro y crédito frente a la aplicación de las NIIF. Caso cooperativas del Valle del Cauca. Cuadernos de Contabilidad, v. 14, n. 36, p. 881-901, 2013.

ROSSÉS, G. F; FERREIRA, G, M, V; STECCA, J, P; GELATTI, C, B. Sistema de gestão em cooperativas: o caso da Cooperativa Agropecuária Júlio de Castilhos. Revista em Agronegócio e Meio Ambiente, v 4, n. 3, Maringá, mar. 2011.

ROYER, A.; BIJMAN, J.; ABEBE, G. K. Cooperatives, partnerships and the challenges of quality upgrading: A case study from Ethiopia. Journal of Co-operative Organization and Management, v. 5, n.1, p. 48-55, mai. 2017.

SECRETARIA DE ESTADO DE MEIO AMBIENTE E DESENVOLVIMENTO ECONÔMICO (SEMADE). 2016. Produto Interno Bruto Estadual 2010-2014. Disponível em: http://www.semade.ms.gov.br/wpcontent/uploads/sites/20/2016/11/PIB-MS2010-2014.pdf. Acesso em: 20 jun. 2017.

SILVA, J. P. Análise financeira das empresas. 12 ed. São Paulo: Atlas, 2013.

SILVA, L. R. et al. Construção de um índice-padrão e análise da performance financeira das empresas de capital aberto que atuam no setor de exploração de Rodovias. Scientia Plena, v. 11, n. 3, mar. 2015.

TAKAMATSU, R. T.; LAMOUNIER, W. M. A Importância da Atualização Monetária de Valores para a Análise das Demonstrações Financeiras. Contabilidade Vista \& Revista, v. 17, n. 2, p. 67-87, abr./ jun. 2006.

ZANELlA, L. C. H. Metodologia de Estudo e de Pesquisa em Administração. Florianópolis: Departamento de Ciências da Administração, UFSC, 2009. 